\title{
Clinicopathological correlation of transient acantholytic dermatosis: A case report
}

\author{
ILARIE BRIHAN ${ }^{1}$, GYULA LÁSZLÓ FEKETE ${ }^{2}$, CONSTANTA TURDA ${ }^{3}$, \\ OVIDIU TICA ${ }^{4}$, ALINA CRISTIANA VENTER ${ }^{4}$, SIMONA IANOSI ${ }^{5}$, \\ CARMEN-DANIELA NEAGOE $^{6}$ and DACIANA ELENA BRANISTEANU ${ }^{7}$
}

\begin{abstract}
${ }^{1}$ Dermatology Department, Dermatology Clinic, Faculty of Medicine and Pharmacy, University of Oradea, 410073 Oradea; ${ }^{2}$ Dermatology Department, Dermatology Clinic, 'George Emil Palade' University of Medicine, Pharmacy, Science and Technology, 540530 Targu Mureș; Departments of ${ }^{3}$ Psycho-Neuroscience and Recovery and ${ }^{4}$ Morphological Disciplines, Faculty of Medicine and Pharmacy, University of Oradea, 410073 Oradea; Departments of ${ }^{5}$ Dermatology and ${ }^{6}$ Internal Medicine, University of Medicine and Pharmacy, 200349 Craiova; ${ }^{7}$ Department of Dermatology, University of Medicine and Pharmacy 'Grigore T. Popa', 700115 Iasi, Romania
\end{abstract}

Received September 13, 2021; Accepted October 13, 2021

DOI: $10.3892 / \mathrm{etm} .2021 .11096$

\begin{abstract}
Transient acantholytic dermatosis (TAD) is a benign, non-familial, non-immune mediated acantholytic disorder of unknown etiology. The presence of polymorphous, unorganized, pruritic lesions on the trunk, associated with focal acantholysis and dyskeratosis, resembles a wide variety of dermatoses. The etiology of TAD (also known as Grover's disease) is unknown, and the success of treatment relies on the correct identification of the disease; however, some cases are refractory to all forms of therapy. For accurate diagnosis, a comprehensive literature review is required. Here, the case of a 55-year-old male with TAD displaying a Darier-like histopathological pattern was reported. The patient was successfully treated with retinoids and acitretin (Neotigason), as well as dapsone, an anti-inflammatory agent, as maintenance therapy. The presence of more than two histological findings, limited to small foci and clinical information, can diagnose Darier disease. The exact pathogenesis has not been elucidated, thus further studies of the pathogenesis of TAD are required.
\end{abstract}

Correspondence to: Dr Constanta Turda, Department of Psycho-Neuroscience and Recovery, Faculty of Medicine and Pharmacy, University of Oradea, Piața 1 Decembrie 10, 410073 Oradea, Romania

E-mail: cturda2003@yahoo.com

Dr Gyula László Fekete, Dermatology Department, Dermatology Clinic, 'George Emil Palade' University of Medicine, Pharmacy, Science and Technology, Strada Gheorghe Doja 12, 540530 Targu Mures, Romania

E-mail: dermafek@yahoo.com

Key words: transient acantholytic dermatosis, clinicopathologic, histologically, pathogenesis, treatment, dapsone

\section{Introduction}

Transient acantholytic dermatosis (TAD), also known as Grover's disease, was named after an individual who thoroughly characterized the disease in 1970 (1). TAD is characterized by papulovesicular eruptions on the trunk, which are typically focal acantholysis and dyskeratosis on histological examination. Characteristic skin lesions are erythematous to red-brown keratotic papules that are localized on the anterior part of the chest, upper part of the back and lower part of the rib cage with widespread scatter and no tendency to coalesce, whereas the scalp, palms and soles are usually not affected (2). Acneiform, vesicular, pustular and rarely bullous lesions, as well as unusual distributions, including unilateral or zosteriform eruptions, have been reported as dermatological presentations of TAD (3). Lesions may resolve with postinflammatory pigmentation. No systemic symptoms are associated with TAD, but oral lesions can develop as slightly painful aphthae. Skin lesions are accompanied by pruritus, with variable degrees of itching from mild to severe. Patients with severe pruritus typically present with multiple, disseminated lesions affecting the neck, shoulders, trunk, arms and legs $(4,5)$.

The presentation of TAD can be subtle or may closely resemble other pruritic dermatoses. TAD is a reactive skin condition that is benign, self-limited and resolves over a period of weeks or months, but there are also cases with long evolution and a tendency to become persistent, which are difficult to manage due to the recurrence of physical signs and persistent pruritus (6). The exact etiology of TAD is unknown.

Viral, bacterial and other pathogens have been proposed as causative agents of TAD, but no causative role has been established. Bullous impetigo, common impetigo, staphylococcal scalded skin syndrome and herpes simplex virus $(\mathrm{HSV}) /$ varicella-zoster virus (VZV) infection result in vesicles that clinically present as bullae and acantholysis on histopathological examination (7). In these cases, for the 
confirmation of the diagnosis, the clinicopathological correlations, epidemiological context and drug intake are necessary. Herpetic infections, including human herpesvirus (HHV)-1, HHV-2 and HHV-3 (also known as VZV), are characterized by an intraepidermal blister, ballooning degeneration of keratinocytes and multinucleate keratinocyte giant cells that allow for easy diagnosis. Epithelial multinucleated giant cells are usually associated with HSV infections, but they also can be present in skin biopsy specimens of benign and malignant skin tumors or in acantholytic dermatoses (8). In special cases, when a herpes simplex infection is suspected, but the skin lesions do not have the typical appearance (for example vesicular lesions at a distance or disseminated vesicles) and when there is a lack of response to antiviral therapy, laboratory tests are performed to rule out the diagnosis of viral infection; the existence of an acantholytic dermatosis must be considered and a skin biopsy must be performed (8).

Heat, sweating and the obstruction of sweat ducts have been suggested as potential causative factors for the disease; however, it has also been reported that xerosis is a potential cause of TAD, and that the disease is exacerbated by cold weather. The disease seems to occur more frequently in patients with atopic dermatitis and asteatotic dermatitis. We hypothesize that the appearance of cutaneous lesions is closely related to individual-specific neurovegetative reactions to cholinergic and adrenergic stimuli, as sweat secretion is regulated by the central nervous system and the autonomic nervous system. Both cholinergic and adrenergic pathways seem to be involved in sweating. Acetylcholine, a neurotransmitter released by sympathetic nerve, acts as a potent stimulator for sweat secretion (9-11). Dysfunction of the autonomic nervous system, in particular the sympathetic nervous system, may cause sweating disorders, such as hyperhidrosis or hypohidrosis (12). The role of sweat gland secretion is to regulate the body temperature when it rises, which is controlled by the sympathetic nervous system (12).

In this context of sweating disorders, drugs and their adverse reactions should be considered. For example, b blockers, which induce hyperhidrosis secondary to $\beta$-adrenoreceptor blockade, are widely used in the treatment of common diseases, such as hypertension and heart failure, that are often encountered in elderly people (11).

Exposure of the normal epidermis to heat (cholinergic stimulation), stress, intense physical exertion, certain professional activities and emotional factors results in increased sweat secretion. However, blocking the acrosyringial ducts may also be a factor contributing to the disruption of sweat secretion. Cold exposure of an intact xerotic epidermis with low sweat secretion, based on atopic dermatitis and asteatotic dermatitis, may be a causative agent of TAD $(10,12)$. In atopic skin, the altered composition of perspiration (concentration of salt, water, lactic acid and urea) may serve a role in the etiopathology of TAD, regardless of the influence of environmental factors on the intensity of perspiration (10). Thyroid dysfunction and neurovegetative abnormalities may also serve important roles in the etiology of TAD.

TAD most commonly affects middle-aged white male patients over the age of 40, but the disease has also been reported in children. Epidemiological studies demonstrated that men are more affected than women, with an incidence ratio of 3:1 (1).
A number of studies have reported a direct relationship between autoimmune antibodies and TAD, but whether the autoimmune antibodies cause TAD or an increase in the number of autoimmune antibodies is a result of TAD is not completely understood $(13,14)$. Therefore, further studies are required to determine whether TAD is an immunological disorder.

The disease is associated with a number of other skin diseases, including eczema, solar keratosis, psoriasis, autoimmune disorders (such as bullous pemphigoid, vitiligo and alopecia areata), immunological diseases and malignancies $(13,15)$. Future studies should assess the cellular and humoral immunity disturbances in TAD, and also investigate its association with the aforementioned skin diseases using a large patient population.

TAD has also been observed in oncology patients. TAD was primarily observed in patients with hematologic malignancies, including acute and chronic myelogenous leukemia and lymphoma, solid tumors and carcinoma of the genitourinary organs $(16,17)$. In previous case reports, it has also been reported that the occurrence of TAD coincided with the onset of malignancy in some cases or with the recurrence of malignancy in three individuals (12\%). In the other 23 oncology patients, TAD was considered to be secondary to specific antineoplastic therapy or excessive perspiration, fever, occlusive immobility, or ionizing or UV radiation (16-18).

Melanoma therapy with cytotoxic T-lymphocyte-associated protein 4 inhibition has been also associated with TAD (18). However, there is not a sufficient number of related cases to consider the disease as a paraneoplastic disorder.

The prevalence of TAD is not available primarily due to the clinical and histopathological similarities with other types of dermatitis, especially with the limited papulovesicular lesions of the trunk. It has been proposed that the disease is under diagnosed. Both the dermatologist and dermatopathologist must be aware of entities that mimic classic acantholytic dermatoses and of rare disease variants, which are characterized by acantholysis. Positive diagnosis is obtained by clinical presentation and histopathological examination, which typically reveals focal acantholysis and dyskeratosis, and spongiosis is also commonly observed. The presence of spongiosis, acantholysis and vesicle formation in the same specimen should raise the possibility of TAD (19).

Acantholysis, associated with dyskeratosis, may mimic Darier disease, Hailey-Hailey disease or pemphigus, and accurate histological examination is important for diagnosis (6). Dermoscopic examination is also limited, as no specific features for TAD have been reported yet. Moreover, immunofluorescence has been proven negative in most cases, as well as inconsistent, and has not yet been directly correlated to the histology of the disease (20); however, this may become an area for research into the etiology of TAD.

From a clinical and histological point of view, the disease resembles numerous dermatoses, including Darier disease, pemphigus foliaceus, Hailey-Hailey disease and dermatitis herpetiformis. Therefore, an accurate diagnosis is difficult as specific lesions occur more frequently in patients with atopic dermatitis and asteatotic dermatitis. Positive diagnosis is based on historical, clinical and histological examination, but 
is difficult because the disease can mimic a lot of dermatoses, especially when it is accompanied by pruritus $(19,21)$.

Treatment success relies on the correct identification of the disease, follow-up over time and multiple checks to prevent recurrences, which appear frequently for refractory cases. Current treatment strategies include topical steroids, calcineurin inhibitors, vitamin D analogs, retinoids, methotrexate and 5-aminolevulinic acid photodynamic therapy. In addition, activities that cause excess heat and sweating should be avoided. Potent topical corticosteroids are used for their anti-inflammatory effect, as well for the control of itching. For pruritus, menthol or pramoxine-containing lotions can also be used (6). Liquid nitrogen therapy has been reported as an effective strategy for patients with persistent TAD, which involves the application of liquid nitrogen on papular lesions for $15 \mathrm{sec}$ beyond formation of the ice field, followed by a second cycle after 3-5 min of complete thawing. At 3 months post-liquid nitrogen therapy, no new lesions or pruritus were observed, only a few residual hyperpigmentation macules were identified and no recurrence within 1 year was reported (22). Vitamin A (50,000 U, 3 times/day for 2 weeks followed by a single daily dose for up to 12 weeks) and isotretinoin (40 mg/day for 2-12 weeks) have been suggested as effective treatment strategies for refractory cases (23). Oral corticosteroids, UV-B exposure, psoralen plus ultraviolet A light, grenz radiation and methotrexate have also been reported to be effective in treating severely resistant cases. However, some cases are refractory to all forms of therapy.

\section{Case report}

A 55 year-old Caucasian male presented in the Dermatology Department (County Emergency Clinical Hospital Oradea, Romania) in July 2018, for well-delimitated, axillary, bilateral erythematous plaques and placards with geographical borders, as well as vesicle-bullae in periphery, some of which were pustulated. Papulovesicles were present at distance on the anterior part of the neck (Fig. 1) and the lateral part of the trunk, where red-brown papular lesions and non-follicular lesions were also observed (Fig. 2). No lesions were observed on the face, scalp, extremities, palms, soles or mucous membranes. The lesions were accompanied by mild pruritus. History revealed no systemic disorders, dermatological diseases or drug intake of medications associated with disease etiology (including anastrozole, vemurafenib, dabrafenib, cetuximab, mercury and d-penicillamine). Papular drug eruption exhibits papules that tend to coalesce into plaques, but do not spare the extremities. The laboratory tests reported nothing pathological, with negative tests for VZV, HSV1 and HSV2. A skin biopsy was performed from a papulovesicle lesion of the trunk. The sample was fixed in $10 \%$ buffered formalin for $24 \mathrm{~h}$ at room temperature. Following paraffin embedding, the tissues were cut into $4-\mu \mathrm{m}$ thick sections, which were then stained with H\&E. Images of the stained sections, obtained with a light microscope, were captured using a DM1000 installed camera (Leica Microsystems, Inc.).

Gross examination revealed a $6 / 4 \mathrm{~mm}$ post-fixation skin specimen with a centrally located vesicle. A polymorphous lesion with suprabasilar and subcorneal spongiosis covered with a thick keratin layer filled with neutrophils was observed

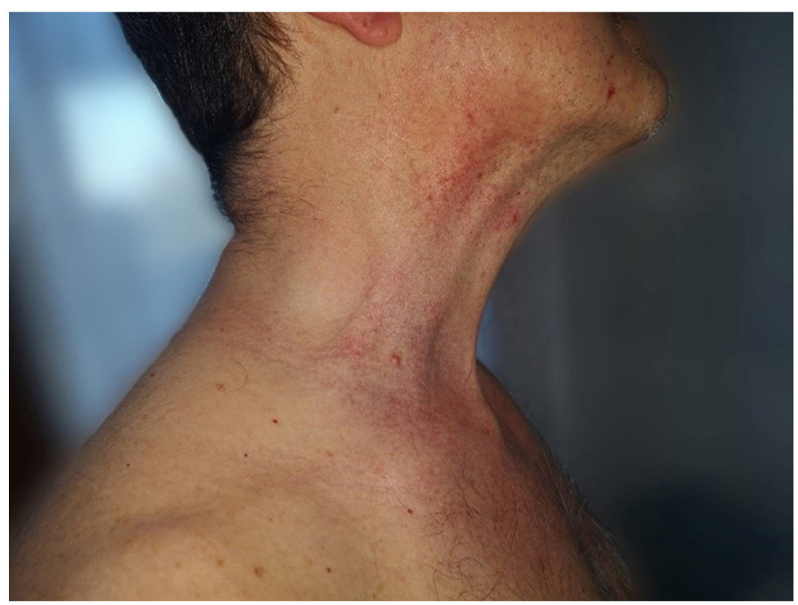

Figure 1. Clinical presentation of erythematous plaques and placards, with vesicle-bullae in the periphery, located on the anterior part of the neck.

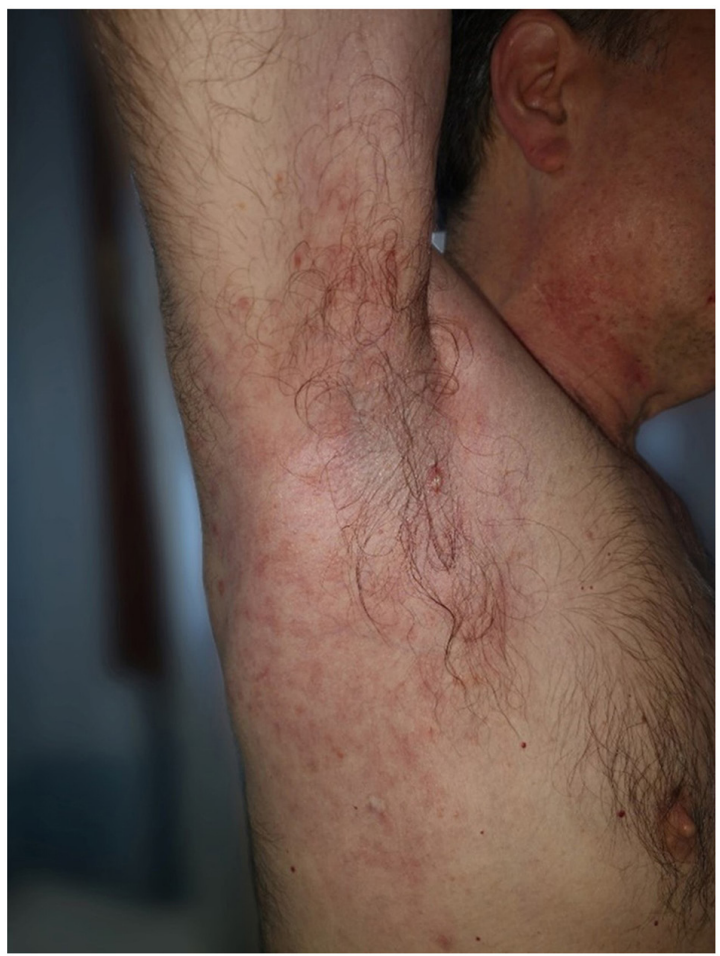

Figure 2. Clinical presentation of red-brown papular lesions and non-follicular lesions located on the lateral part of the trunk.

(Figs. 3 and 4). In one area, there was an erosion of the epidermis, potentially developed secondary to scratching. As shown in Fig. 5, a neutrophil microabscess adjacent to this erosion was identified. In the papillary dermis, there was edema and mixed perivascular inflammation (neutrophils, lymphocytes, plasma cells and eosinophils). Detailed examination of the spongiosis revealed some dyskeratotic round cells, with abundant granules of keratohyalin associated with elongation of rete ridges (Darier-like patterns). The superficial dermis that contains perivascular lymphocytes and severe edema is shown in Fig. 5. A total of 13 levels of the tissue sections were needed for diagnosis of the disease via H\&E staining (no immunofluorescence staining was performed). 


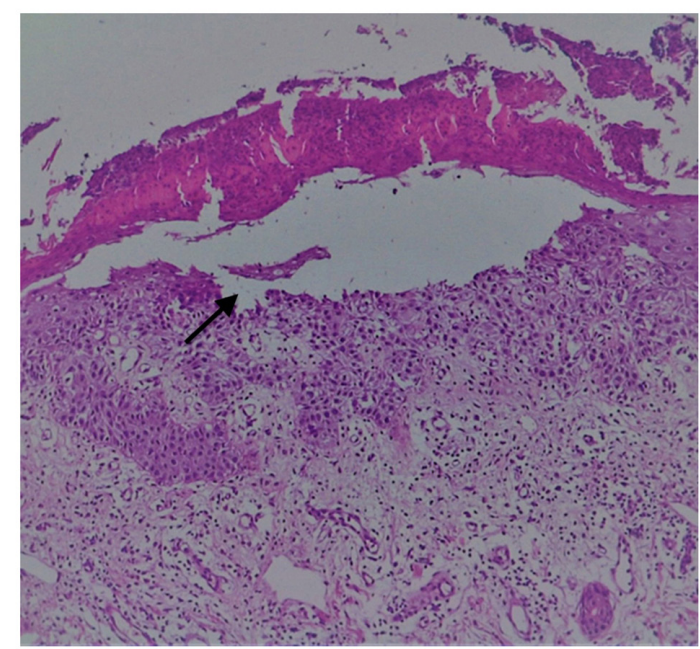

Figure 3. H\&E staining of suprabasilar and subcorneal acantholysis (arrow). Magnification, x10.

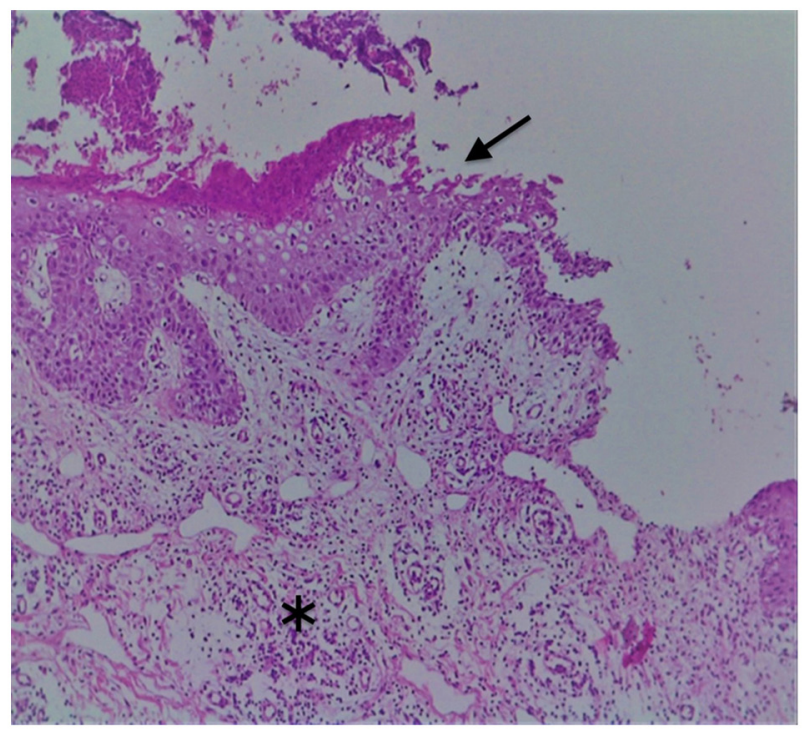

Figure 4. H\&E staining of suprabasilar acantholysis (arrow) with a brisk dermal mixed inflammation (asterisk). Magnification, x10.

The clue for positive diagnosis in this case was the multiplicity of histological lesions in a single papulovesicle of only several rete ridges wide. There were no acantholytic cells in the lesion, which excluded the possibility of a pemphigus vulgaris-like lesion in this patient, but suprabasilar spongiosis can be associated with other pemphigus-like lesions. A presence of $>2$ histological findings limited to small foci and specific clinical information could mislead towards the diagnosis of Darier disease.

\section{Discussion}

Bilateral axillaries erythematous plaques that are well delimitated with geographical borders and pustules in the periphery, suggested tinea corporis, but the coexistence of polymorphous lesions as bullas, papule-vesicles, and red-brown papular lesions on the anterior part of the neck and on the lateral part

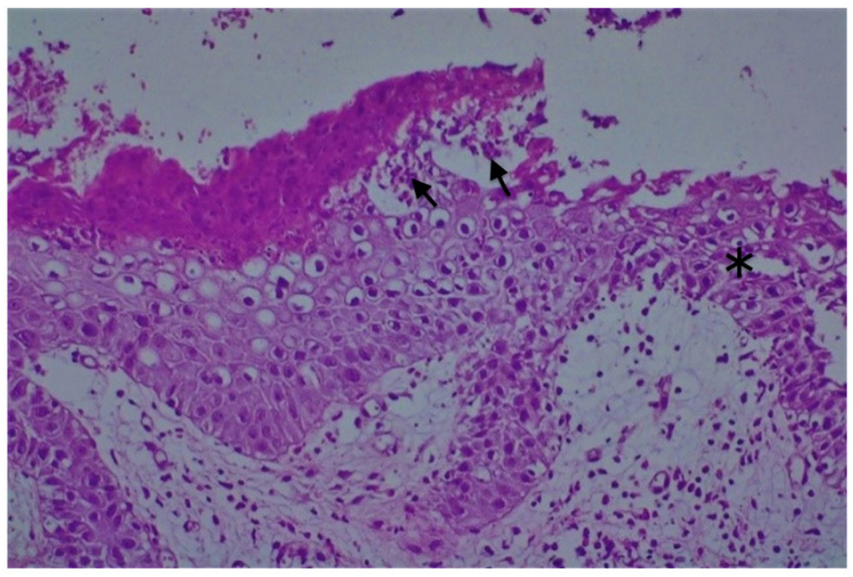

Figure 5. H\&E staining of intraepidermal spongiosis (asterisk) with neutrophil microabscesses (arrows). Magnification, x20.

of the trunk were considered elements for a complex diagnosis and the decision for a skin biopsy was made in the present case.

The second option was familial benign pemphigus (Hailey-Hailey Disease), considering the vesicles and erythematous plaques, erosions located on the axillary areas, bilateral, chest and neck, but in the case assessed in the present study, there was no family history of this disease. Hailey-Hailey Disease is a chronic autosomal dominant disorder with incomplete penetrance. Among those with familial benign pemphigus, $\sim 2 / 3$ of patients have a family history of the disorder. Also, in cases of familial benign pemphigus, vesicles and erythematous plaques with overlying crusts typically occur in the genital area. Histological findings in familial benign pemphigus are suprabasilar and widespread acantholysis (6), not a focal one with spongiosis as seen in the present case.

From a histological point of view, TAD presents as focal acantholysis frequently associated with dyskeratosis that may mimic Darier disease, Hailey-Hailey disease or pemphigus. Darier disease (keratosis follicularis) is histologically characterized by two primary features, acantholysis and dyskeratosis. Hyperkeratosis, a keratin plug that overlies each lesion, is also common. Keratosis follicularis, also known as Darier disease or Darier-White disease, is an autosomal dominantly-inherited genodermatosis that is characterized by greasy hyperkeratotic papules in seborrheic regions, nail abnormalities and changes to the mucous membrane. The majority of patients $(80 \%)$ have mild flexural involvement, with scattered papules in the groin, axillae or sub mammary skin in women. However, in $10 \%$ of patients, flexural disease predominates with large, warty, vegetative plaques in the axillae, groin or perineum (5).

A lack of history of Darier Disease, no keratotic warty lesions on seborrheic areas, no follicular involvement or abnormalities in the nails and mucous membranes resulted in the exclusion of the Darier disease in the present case despite histological similarities.

TAD is an acquired acantholytic dermatosis, not an inherited acantholytic dermatitis, such as Darier disease and Hailey-Hailey disease. Plaques with vesicles, erosions and erythematous papules are also seen in dermatitis herpetiformis (24); however, the present case did not present with 
clustered vesicles, herpetiform pattern distributed over elbows, knees and/or buttocks. The diagnosis of dermatitis herpetiformis is suspected based on the distribution of the eruption and the genetic predisposition to the development of gluten sensitivity, and the disease is generally accepted as a cutaneous manifestation of celiac disease. Biopsy specimens of lesional skin reveals neutrophils in the dermal papillae, with the formation of microabscesses that progress to subepidermal vacuolization and vesicle formation. Edema and eosinophils can also be present. Granular IgA deposits observed in the dermal papillae of perilesional skin via direct immunofluorescence is the criterion standard of diagnosis $(6,24)$.

In the present case, there was no history or evidence for gluten-sensitive enteropathy. In the skin specimen biopsy, papillary dermis edema and mixed perivascular inflammation, containing neutrophils, lymphocytes, plasma cells and eosinophils, were observed, but instead of a subepidermal vesicle, a supra-epidermic, centrally located vesicle, a polymorphous lesion with suprabasilar and subcorneal spongiosis associated with acantholysis and dyskeratosis was identified.

Pemphigus foliaceus, which is characterized by intraepidermal acantholysis and the loss of intercellular adhesion of keratinocytes in the upper parts of the epidermis resulting in the formation of superficial blisters, could be another potential diagnosis. From a clinical point of view, typical pemphigus foliaceus presents as small, superficial blisters or flaccid bullas on an erythematosus base, primarily confined to seborrheic areas, which are difficult to find because they are transient and transform fast into crusted, scaly erosions. Pemphigus foliaceus is an autoimmune vesiculobullous disorder that is characterized by intraepidermal acantholysis, intercellular deposition of IgG and complement on mucocutaneous junctions, and circulating autoantibodies (6).

In the present case, there was no involvement of the face and scalp, nor any itchy papules on the trunk. Only a few erosions were identified on the trunk, not as primary elementary lesions, but were considered secondary to scratches. Suprabasilar acantholysis, associated with diskeratosis round cell spongiosis with keratohyalin granules, was also highlighted.

Galli-Galli disease is a rare variant of the genodermatosis Dowling-Degos disease, with clinical and histological features similar to TAD (25), belonging to the spectrum of reticulate pigment dermatoses, classified as an acantholytic variant of Dowling-Degos disease on the basis of its characteristic clinical and histological findings. It is a rare inherited condition, characterized by $1-2 \mathrm{~mm}$ slightly keratotic red to dark brown papules, which are confluent in a reticulate pattern, slowly progressive and result in disfiguring reticulate hyperpigmentation of the flexures. The histopathological characteristics consist of suprabasal and non-dyskeratotic acantholysis.

In the present case, there was no evidence of inherited disorders, and no clinical signs for keratotic papules or reticulate hyperpigmentation. The patient presented with papulovesicular lesions on an erythematous base in bilateral axillae, and the skin specimen biopsy from a papulovesicle lesion of the trunk showed suprabasal acantholysis with dyskeratosis and spongiosis.

Our final diagnosis was TAD based on the clinical and histological findings.
Oral treatment with retinoids and acitretin (Neotigason) ( $20 \mathrm{mg} / \mathrm{day}$ for 9 months) was initiated. The treatment displayed good results, and dapsone, an anti-inflammatory agent related to inhibition of neutrophil migration and function, was administered as a maintenance therapy to avoid recurrences. The treatment was selected based on the existence of mixed perivascular inflammation in papillary dermis, including neutrophils, lymphocytes, plasma cells and eosinophils, which were identified by histological examination.

Dapsone is an aniline derivate belonging to the group of synthetic sulfones with antimicrobial, antiprotozoal and anti-inflammatory effects that resemble non-steroidal anti-inflammatory drugs. The underlying mechanism of action of dapsone is not completely understood. Previous studies showed that dapsone inhibits chemotaxis when certain stimuli are applied, and displays an inhibitory effect on prostaglandin synthesis and liberation and on eosinophil peroxidase, which provides a potential explanation for the therapeutic efficacy of dapsone in eosinophil-mediated diseases $(26,27)$. The disease-specific antiphlogistic and antichemotactic activities and steroid-sparing effects of dapsone have resulted in its use for the treatment of neutrophilic and/or eosinophilic dermatoses in chronic disorders, in and outside the field of dermatology (e.g. bronchial asthma) $(26,27)$. The patient was monitored during therapy for well-known adverse effects of dapsone, including hematological effects (e.g. anemia, met-Hb formation, hemolysis and agranulocytosis), adverse gastrointestinal effects (e.g. anorexia, abdominal pain, nausea and vomiting), hepatic dysfunction and neuropathy. Good results obtained with long-term dapsone treatment resulted in selecting this treatment option for maintenance therapy to prevent recurrences.

TAD is underdiagnosed due to its resemblance to a number of other dermatoses, including inherited dermatoses (such as Darier Disease, Hailey-Hailey disease and Galli-Galli disease) and immunological-mediated dermatoses (such as pemphigus foliaceus, IgA pemphigus and dermatitis herpetiformis). Due to the clinical similarities with other dermatoses and variable histopathologic findings, a high index of suspicion is necessary for the accurate diagnosis of this disease, and a clinicopathologic correlation is required.

The exact pathogenesis of TAD has not yet been elucidated. Variations in sweat responses to environmental factors, stress and emotions may be important causative factors. Therefore, the disease could be considered as polypathogenic in nature. Future studies are required to further investigate the etiology of this disease.

\section{Acknowledgements}

Not applicable.

\section{Funding}

No funding was received.

\section{Availability of data and materials}

All data generated or analyzed during this study are included in this published article. 


\section{Authors' contributions}

IB was responsible for the clinical management of the patient, and the processing and scientific interpretation of the data. GLF was responsible for evaluating the data and revising the manuscript. CT was responsible for the analysis of the specialized literature, the study of the clinical case and writing the manuscript. OT was responsible for obtaining and processing histopathological data. ACV was responsible for data processing and revising the manuscript. SI was responsible for studying, data processing and revising the manuscript. CDN was responsible for the analysis of the specialized literature and interpreting the data. DEB was responsible for evaluating the data and revising the manuscript. IB, CT and GLF confirm the authenticity of all the raw data. All authors read and approved the final manuscript.

\section{Ethics approval and consent to participate}

Written informed consent was obtained from the patient.

\section{Patient consent for publication}

Written informed consent was obtained from the patient.

\section{Competing interests}

The authors declare that they have no competing interests.

\section{Authors' information}

Ilarie Brihan is the Head of the Dermatology Clinic at County Emergency Clinical Hospital Oradea.

\section{References}

1. Grover RW: Transient acantholytic dermatosis. Arch Dermatol 101: 426-434, 1970.

2. Fantini F, Kovacs E and Scarabello A: Unilateral transient acantholytic dermatosis (Grover's disease) along Blaschko lines. J Am Acad Dermatol 47: 319-320, 2002.

3. Liss WA and Norins AL: Zosteriform transient acantholytic dermatosis. J Am Acad Dermatol 29: 797-798, 1993.

4. Kanzaki T and Hashimoto K: Transient acantholytic dermatosis with involvement of oral mucosa. J Cutan Pathol 5: 23-30, 1978

5. Brown-Joel ZO, Chung J and Stone MS: Pityriasis rubra pilaris-like eruption in the setting of transient acantholytic dermatosis. JAAD Case Rep 5: 733-735, 2019.

6. Miller JL and Hurley HJ: Diseases of the eccrine and apocrine sweat glands. In: Dermatology. Bolognia JL, Jorizzo JL and Rapini PR (eds). Vol. 1. Mosby Elsevier, London, p543, 2007.

7. Ho J and Bhawan J: Mimickers of classic acantholytic diseases. J Dermatol 44: 232-242, 2017.
8. Cohen PR, Paravar T and Lee RA: Epidermal multinucleated giant cells are not always a histopathologic clue to a herpes virus infection: Multinucleated epithelial giant cells in the epidermis of lesional skin biopsies from patients with acantholytic dermatoses can histologically mimic a herpes virus infection. Dermatol Pract Concept 4: 21-27, 2014.

9. Scheinfeld N and Mones J: Seasonal variation of transient acantholytic dyskeratosis (Grover's disease). J Am Acad Dermatol 55: 263-268, 2006.

10. Hendricks AJ, Vaughn AR, Clark AK, Yosipovitch G and Shi VY: Sweat mechanisms and dysfunctions in atopic dermatitis. J Dermatol Sci 89: 105-111, 2018.

11. Tatu AL, Elisei AM, Chioncel V, Miulescu M and Nwabudike LC: Immunologic adverse reactions of $\beta$-blockers and the skin. Exp Ther Med 18: 955-959, 2019.

12. Hu Y, Converse C, Lyons MC and Hsu WH: Neural control of sweat secretion: A review. Br J Dermatol 178: 1246-1256, 2018.

13. Phillips C, Kalantari-Dehaghi M, Marchenko S, Chernyavsky AI, Galitovskiy V, Gindi V, Chun S, Paslin D and Grando SA: Is Grover's disease an autoimmune dermatosis? Exp Dermatol 22: 781-784, 2013.

14. Ellenbogen E, Geller S, Azrielant S, Zeeli T, Goldberg I, Schmidt E, Zillikens D, Mrowietz U, Sherman S, Mercer S, et al: Grover disease and bullous pemphigoid: A clinicopathological study of six cases. Clin Exp Dermatol 44: 524-527, 2019.

15. Quirk CJ and Heenan PJ: Grover's disease: 34 Years on. Australas J Dermatol 45: 83-88, 2004.

16. Ishibashi M, Nagasaka $T$ and Chen KR: Remission of transient acantholytic dermatosis after the treatment with rituximab for follicular lymphoma. Clin Exp Dermatol 33: 206-207, 2008.

17. Fujita Y, Sato-Matsumura KC and Ohnishi K: Transient acantholytic dermatosis associated with B symptoms of follicular lymphoma. Clin Exp Dermatol 32: 752-754, 2007.

18. Guana AL and Cohen PR: Transient acantholytic dermatosis in oncology patients. J Clin Oncol 12: 1703-1709, 1994.

19. Fernández-Figueras MT, Puig L, Cannata P, Cuatrecases M, Quer A, Ferrándiz C and Ariza A: Grover disease: A reappraisal of histopathological diagnostic criteria in 120 cases. Am J Dermatopathol 32: 541-549, 2010.

20. Millns JL, Doyle JA and Muller SA: Positive cutaneous immunofluorescence in Grover's disease. Arch Dermatol 116: 515, 1980.

21. Lacarrubba F, Boscaglia S, Nasca MR, Caltabiano R and Micali G: Grover's disease: Dermoscopy, reflectance confocal microscopy and histopathological correlation. Dermatol Pract Concept 7: 51-54, 2017.

22. Galamgam J and Lee D: Treatment of transient acantholytic dermatosis with liquid nitrogen. JAAD Case Rep 6: 341-343, 2020.

23. Helfman RJ: Grover's disease treated with isotretinoin. Report of four cases. J Am Acad Dermatol 12: 981-984, 1985.

24. Plotnikova N and Miller JL: Dermatitis herpetiformis. Skin Therapy Lett 18: 1-3, 2013.

25. Gilchrist H, Jackson S, Morse L, Nicotri T and Nesbitt LT: Galli-Galli disease: A case report with review of the literature. J Am Acad Dermatol 58: 299-302, 2008.

26. Wozel G and Blasum C: Dapsone in dermatology and beyond. Arch Dermatol Res 306: 103-124, 2014.

27. Debol SM, Herron MJ and Nelson RD: Anti-inflammatory action of dapsone: inhibition of neutrophil adherence is associated with inhibition of chemoattractant-induced signal transduction. J Leukoc Biol 62: 827-836, 1997.

This work is licensed under a Creative Commons Attribution-NonCommercial-NoDerivatives 4.0 International (CC BY-NC-ND 4.0) License. 